PHYSICAL REVIEW E 93, 029901(E) (2016)

\title{
Publisher's Note: Local-time representation of path integrals [Phys. Rev. E 92, 062137 (2015)]
}

Petr Jizba and Václav Zatloukal

(Received 29 January 2016; published 12 February 2016)

DOI: 10.1103/PhysRevE.93.029901

This paper was published online on 22 December 2015 with an error in the author affiliations. The author affiliations should read as

Petr Jizba ${ }^{1,2, *}$ and Václav Zatloukal ${ }^{2,3, \dagger}$

${ }^{1}$ ITP, Freie Universität in Berlin, Arnimallee 14, D-14195 Berlin, Germany

${ }^{2}$ FNSPE, Czech Technical University in Prague, Břehová 7, 11519 Praha 1, Czech Republic

${ }^{3}$ Max Planck Institute for the History of Science, Boltzmannstrasse 22, D-14195 Berlin, Germany

The paper has been corrected as of 29 January 2016. The affiliations are incorrect in the printed version of the journal. 